\title{
Tensor DoA Estimation with Directional Elements
}

\author{
Francesca E. D. Raimondi, Pierre Comon
}

\begin{abstract}
Directivity gain patterns are treated as a physical diversity for tensor array processing, replacing space diversity, in addition to time and space shift diversities. We show that the tensor formulation allows to estimate Directions of Arrival (DoAs) under the assumption of unknown gain patterns, improving the performance of the omnidirectional case. We propose a trilinear model where one dimension of the multi-way data array is fully provided by gain patterns, allowing tensor approaches even when space diversity is missing due to sensor overlap.
\end{abstract}

Index Terms-DoA estimation, Tensor, Directivity, Gain, Patterns, Smart Antennas, Directional Arrays

\section{INTRODUCTION}

D IRECTION of Arrival (DoA) estimation is a central problem in array signal processing, e.g. for telecommunications, seismology, speech, biomedical engineering. Acquisition systems are composed of multiple sensors that receive source signals from different directions [1], [2]. Directional sensor arrays have been used in the context of smart antennas, through beamforming techniques [3] and null-steering (see [4], [5] for a list of examples). Several studies have investigated directional elements for DoA estimation, such as [6], [7], both with Uniform Circular Arrays (UCA). High resolution DoA estimation through the MUSIC algorithm [8] with known sensor gains has been further studied in [5], where the effects and advantages of different directivity patterns have been considered, as well as a realistic dipole array implementation; a derivation of the Cramér-Rao Bound (CRB) for directional elements of a UCA is also included.

If sensors are omnidirectional (as in most of the literature in array processing), only relative phase differences between sensors are needed to extract DoA information. However, if the antenna elements are directional, one may jointly exploit gain and phase differences in every direction of interest. To our knowledge, existing studies on DoA estimation in the presence of directional elements mostly cover the case of known directivity gains; conversely, our aim is to treat the case of DoA estimation using sensors with unknown gain patterns.

Tensor analysis has been applied to sensor arrays for DoA estimation, which requires at least three physical diversities, such as time, space and space shift [9], [10] through multiple translated subarrays. Recently, a general formulation of tensor array processing has been extended to the wideband case through multiple physical diversities in [11]. We show in this paper that gain patterns can constitute a diversity in their own right.

In fact, contrary to more standard approaches, e.g. MUSIC [2], [8], tensor approaches can handle unknown gains, as

Francesca E. D. Raimondi and Pierre Comon are with GIPSA-Lab (Department of Image and Signal-processing), CNRS, Université Grenoble Alpes, 38400 Saint Martin d'Hères, France. This work was supported by ERC AdG2013-320594 grant DECODA. subsequently demonstrated. On the other hand, ESPRIT [12] can be seen as a particular tensor approach and can solve the localization problem if two identical translated subarrays are available. However, in [12], gain patterns have not been explicitly taken into account as a pure source of diversity (i.e. when all sensors are co-located within each subarray, and only differ in their directivity). The same observation applies to multi-dimensional extensions of ESPRIT [13], [9]. We show that even when directional sensors completely overlap within a subarray, thus canceling space diversity, their gain patterns allow a trilinear tensor model, fully replacing the space diversity of [9].

More precisely, the rotational invariance of [12] was extended to multiple space shift translations through a subspace fitting approach in [13] for collinear space shifts, and a deterministic tensor approach in [9] for arbitrary space shifts. In this scenario a reference subarray (representing space diversity through sensors located at different positions) is repeated through multiple translations (representing space shift diversity through dislocated subarrays). In [9], [12], [13], gain patterns have not been exploited to improve the estimation performance. Indeed, although they may be unknown, gain patterns contain important spatial information about impinging sources and their inclusion into the model may help the underlying low rank approximation problem.

We propose a trilinear model, where one dimension of the multi-way data array is fully provided by gain patterns, allowing tensor approaches even when space diversity is missing due to sensor overlap. Computer results are reported as a function of SNR, sensor directivity, and sensor overlap, in comparison to Cramér-Rao Bounds. The effect of directivity patterns is also shown with respect to the equivalent omnidirectional case. We think that sensor gain patterns have considerable potential in the context of small electronic devices with limited space available.

\section{Physical Model}

Assume $R$ source signals impinge on an array of $L$ sensors, each located at a position in space defined by a vector $\boldsymbol{p}_{\ell} \in$ $\mathbb{R}^{3}, 1 \leq \ell \leq L$. For each source, denote the angles of arrival by a vector $\theta_{r}=\left[\phi_{r}, \psi_{r}\right]$ in $3 \mathrm{D}, 1 \leq r \leq R$, or by a scalar $\theta_{r}=\phi_{r}$ if we restrict our attention to a localization problem in $2 \mathrm{D}$. We assume that the signal received at the $\ell$-th sensor at time $t, 1 \leq t \leq T$ follows the additive model below:

$$
x_{\ell}(t)=\sum_{r=1}^{R} g_{\ell}\left(\theta_{r}\right) \varsigma_{r}\left(t-\tau_{\ell}\left(\theta_{r}\right)\right)+n_{\ell}(t)
$$

where $\varsigma_{r}(t) \in \mathbb{R}$ is the $r$-th source signal, $\tau_{\ell}$ is the delay of arrival and $n_{\ell}(t)$ refers to white Gaussian noise. In the far field, if $c$ denotes the (constant) propagation speed, we have 
$\tau_{\ell}(\theta)=\boldsymbol{p}_{\ell}^{\top} \boldsymbol{d}(\theta) / c$, where $\boldsymbol{d}(\theta)$ is the unit modulus vector pointing in direction $\theta$.

Notice that in our framework, each sensor may have its own gain pattern $g_{\ell}: \mathbb{R} \longmapsto \mathbb{R}^{+}$. In a narrow band around radial frequency $\omega_{0}$, we can work in baseband and write the complex envelope of received signals as

$$
x_{\ell}(t)=\sum_{r=1}^{R} g_{\ell}\left(\theta_{r}\right) s_{r}(t) e^{-\jmath \omega_{0} \tau_{\ell}\left(\theta_{r}\right)}+n_{\ell}(t)
$$

where $s_{r}(t)$ is the complex envelope of the $r$-th waveform $\varsigma_{r}(t)$ around $\omega_{0}$. Now, define $\boldsymbol{x}(t)=\left[x_{1}(t), \ldots, x_{L}(t)\right]^{T}$ and steering vectors $\boldsymbol{a}\left(\theta_{r}\right) \in \mathbb{C}^{L}$ with entries $a_{\ell}\left(\theta_{r}\right)=$ $g_{\ell}\left(\theta_{r}\right) e^{-\jmath \omega_{0} \tau_{\ell}\left(\theta_{r}\right)}$. This leads to the usual compact form [8]

$$
\boldsymbol{x}(t)=\boldsymbol{A}(\boldsymbol{\theta}) \boldsymbol{s}(t)+\boldsymbol{n}(t) \in \mathbb{C}^{L}
$$

with $\boldsymbol{s}(t)=\left[s_{1}(t), \ldots, s_{R}(t)\right]^{T}, \boldsymbol{n}(t)=\left[n_{1}(t), \ldots, n_{L}(t)\right]^{T}$, $\boldsymbol{\theta}=\left[\theta_{1}, \ldots, \theta_{R}\right]$, and the $L \times R$ steering matrix, $\boldsymbol{A}(\boldsymbol{\theta})=$ $\left[\boldsymbol{a}\left(\theta_{1}\right), \ldots, \boldsymbol{a}\left(\theta_{R}\right)\right]$.

\section{A. Multiple subarrays}

Now, broadening the original idea developed in [9], assume we have at our disposal a set of $M$ subarrays, each containing $L$ directional sensors, and deducible from each other by a translation (see Figure 1). Choose one of these subarrays as a reference, label it with $m=1$, and denote by $\boldsymbol{\delta}_{m}, m>1$ the vectors defining the translations to obtain the $M-1$ remaining subarrays. To simplify subsequent equations, we also define $\boldsymbol{\delta}_{1}=\mathbf{0}$. The delay of arrival of the $r$-th source to reach sensor $\ell$ of subarray $m$ is then $\tau_{\ell}\left(\theta_{r}\right)+\zeta_{m}\left(\theta_{r}\right)$, where $\zeta_{m}\left(\theta_{r}\right)=$ $\boldsymbol{\delta}_{m}^{\top} \boldsymbol{d}\left(\theta_{r}\right) / c$. Then, at fixed radial frequency $\omega_{0}$, the complex envelope of the signals received at the $\ell$-th sensor of the $m$-th subarray can be written as $\mathcal{X}_{\ell m t}=\mathcal{M}_{\ell m t}^{f u l l}+\mathcal{N}_{\ell m t}$, where [11]:

$$
\begin{aligned}
\mathcal{M}_{\ell m t}^{\text {full }}= & \sum_{r=1}^{R} A_{\ell r}^{\text {full }} B_{m r} S_{t r} \in \mathbb{C}^{L \times M \times T} \\
\text { with } & \left\{\begin{array}{l}
A_{\ell r}^{\text {full }}=g_{\ell}\left(\theta_{r}\right) e^{-\jmath \omega_{0} \tau_{\ell}\left(\theta_{r}\right)} \\
B_{m r}=e^{-\jmath \omega_{0} \zeta_{m}\left(\theta_{r}\right)} \\
S_{t r}=s_{r}(t)
\end{array}\right.
\end{aligned}
$$

Notice that the $L \times R$ steering and pattern matrix, $\boldsymbol{A}$, is the same as in $\S$ II. Space shift elements of the $M \times R$ steering matrix, $\boldsymbol{B}$, are a function of the delay of arrival on each subarray $m$. Finally, source complex envelopes constitute the $T \times R$ signal matrix, $\boldsymbol{S}$.

If the acquisition system is composed of $M=2$ subarrays, deduced from each other by a single translation $\delta=\delta_{2}$, the tensor approach based on model (4) reduces to ESPRIT [12]:

$$
\left\{\begin{array}{l}
\boldsymbol{x}_{1}(t)=\boldsymbol{A} \boldsymbol{s}(t)+\boldsymbol{n}_{1}(t) \\
\boldsymbol{x}_{2}(t)=\boldsymbol{\Phi} \boldsymbol{A} \boldsymbol{s}(t)+\boldsymbol{n}_{2}(t)
\end{array}\right.
$$

where $\boldsymbol{\Phi}=\operatorname{Diag}\left\{e^{-\jmath \omega_{0} \zeta\left(\theta_{1}\right)}, \cdots, e^{-\jmath \omega_{0} \zeta\left(\theta_{R}\right)}\right\}$ is a unitary operator that relates both subarrays, and $\zeta\left(\theta_{r}\right)=\boldsymbol{\delta}^{\top} \boldsymbol{d}\left(\theta_{r}\right) / c{ }^{1}$

We shall work under the assumptions summarized below.

${ }^{1}$ If $M=2$, we also need to fix a rotation ambiguity with respect to the translation axis.

\section{Assumptions:}

A1 The first sensor $(\ell=1)$ is taken as origin, i.e., $\boldsymbol{p}_{1}=\mathbf{0}$, and has unit gain in all directions, i.e. $g_{1}(\theta)=1, \forall \theta$.

A2 The first subarray is considered as a reference, i.e., $\boldsymbol{\delta}_{1}=$ $\mathbf{0}$, without restricting the generality.

A3 Space shifts $\boldsymbol{\delta}_{1}, \ldots, \boldsymbol{\delta}_{M}$ are known, whereas the matrix of sensor positions $\boldsymbol{P}=\left[\boldsymbol{p}_{2}, \ldots, \boldsymbol{p}_{L}\right]$ is unknown. ${ }^{2}$

A4 Sensor gains $g_{\ell}(\theta), \ell>1$, are unknown, real (which is actually equivalent to assuming that their phase is known) and frequency-flat.

A5 Sources $s_{r}(t)$ are deterministic.

A6 Wave propagation speed $c$ does not depend on frequency (i.e. the medium is not dispersive).

A7 Noise is circular complex white Gaussian.

Notice that Assumptions A1 and A2 are not restrictive, and permit to fix the scale indeterminacies in model (4), as pointed out in the next section. Assumption A4 means that $L$ continuous real functions are unknown. However, they appear in the model only at values $\theta_{r}$, so that we may consider only the $(L-1) \times R$ matrix, $\boldsymbol{G}$, with $G_{\ell r}=g_{\ell+1}\left(\theta_{r}\right), 1 \leq \ell \leq L-1$, as unknown. The circularity Assumption A7 could be relaxed to the price of an increased notational complexity, as in [10].

1) Diversity of Gain Patterns Only: Notice that if sensors within a subarray do overlap, i.e. are located at the same place, and differ only through their directivity, then $\boldsymbol{p}_{\ell}=0, \forall \ell$, and matrix $\boldsymbol{A}$ only contains information about gain patterns: $A_{\ell r}=$ $g_{\ell}\left(\theta_{r}\right)$. Therefore, in this degenerate case, the approaches of [13], [9] do not apply and the only space information is carried by space-shift matrix $\boldsymbol{B}$ :

$$
\begin{aligned}
& \mathcal{M}_{\ell m t}^{\text {gain }}= \sum_{r=1}^{R} A_{\ell r}^{\text {gain }} B_{m r} S_{t r} \in \mathbb{C}^{L \times M \times T} \\
& \text { with }\left\{\begin{array}{lll}
A_{\ell r}^{\text {gain }} & =g_{\ell}\left(\theta_{r}\right) \\
B_{m r} & =e^{-\jmath \omega_{0} \zeta_{m}\left(\theta_{r}\right)} \\
S_{t r} & = & s_{r}(t)
\end{array}\right.
\end{aligned}
$$

Notice that in this way we managed to decouple space information (that we know through shifts $\boldsymbol{\delta}_{m}$ ) from gain pattern information (that we do not know). Model (5) is the starting point of our contribution.

2) Diversity of Space Shift Only: In this case, $g_{\ell}(\theta)=1$ $\forall \theta, \forall \ell$, and we end up with the classic omnidirectional model described in [9]: $A_{\ell r}^{\text {shift }}=e^{-\jmath \omega_{0} \tau_{\ell}\left(\theta_{r}\right)}$.

\section{B. Tensor Decomposition}

Any tensor represented by an array $\mathcal{M}$ of size $L \times M \times T$ can be expressed as a sum of $R$ decomposable terms: $\mathcal{M}=$ $\sum_{r=1}^{R} \mathcal{D}(r)$ [14]. By decomposable, it is meant that there exist $R$ triplets of vectors $\{\boldsymbol{u}(r), \boldsymbol{v}(r), \boldsymbol{w}(r)\}$ such that $\mathcal{D}_{\ell m t}(r)=$ $u_{\ell}(r) v_{m}(r) w_{t}(r)$, or, equivalently, $\mathcal{D}(r)=\boldsymbol{u}(r) \otimes \boldsymbol{v}(r) \otimes$ $\boldsymbol{w}(r)$, where $\otimes$ denotes outer (tensor) product. When $R$ is minimal, it is called tensor rank, and this decomposition is unique if $R$ is not too large; for instance, $R<L M T /(L+$ $M+T-2)$ is sufficient almost surely [15].

\footnotetext{
${ }^{2}$ This is the standard assumption of ESPRIT approaches [2], [12], [13], [9], i.e. uncalibrated subarrays. However, with unknown space shifts and known sensor positions, the mathematical problem is the same [10].
} 
Hence in the absence of noise, one can identify every term in (4) with a decomposable tensor, that is: $A_{\ell r} B_{m r} S_{t r}=$ $u_{\ell}(r) v_{m}(r) w_{t}(r)$. This identifiability property is the main motivation in resorting to tensor-based algorithms. However, there is still a scaling ambiguity that cannot be resolved, in general. In fact, denote by $\boldsymbol{a}_{r}, \boldsymbol{b}_{r}$ and $\boldsymbol{s}_{r}$ the columns of matrices $\boldsymbol{A}, \boldsymbol{B}$ and $\boldsymbol{S}$, respectively. Even if the entries $\mathcal{D}_{\ell m t}(r)=A_{\ell r} B_{m r} S_{t r}$ are known, or equivalently $\mathcal{D}(r)=$ $\boldsymbol{a}_{r} \otimes \boldsymbol{b}_{r} \otimes \boldsymbol{s}_{r}$, column vectors are only determined up to scaling factors since $\boldsymbol{a}_{r} \otimes \boldsymbol{b}_{r} \otimes \boldsymbol{s}_{r}=\alpha \boldsymbol{a}_{r} \otimes \beta \boldsymbol{b}_{r} \otimes \frac{1}{\alpha \beta} \boldsymbol{s}_{r}$, for any pair of nonzero scalars $(\alpha, \beta)$. In other words, this leaves $2 R$ scalar unknowns for the tensor model

$$
\mathcal{X}=\sum_{r=1}^{R} \boldsymbol{a}_{r} \otimes \boldsymbol{b}_{r} \otimes \boldsymbol{s}_{r}+\mathcal{N}
$$

unless some other constraints are imposed. In the present context, we precisely know that the first entries of $\boldsymbol{a}_{r}$ and $\boldsymbol{b}_{r}$ are equal to 1, $\forall r$, because of Assumptions A1 and A2. These $2 R$ constraints hence completely fix scaling indeterminacies.

\section{A Physical Measure for Identifiability: Coherences}

An angular measure was introduced in [16], in order to assess identifiability conditions easy to compute and interpret. For instance, the coherence $\mu_{A}$ of CPD factor matrix $\boldsymbol{A}=$ $\left[\boldsymbol{a}_{1}, \ldots, \boldsymbol{a}_{R}\right]$ is defined as $\mu_{A}=\max _{p \neq q}\left|\boldsymbol{a}_{p}^{\mathrm{H}} \boldsymbol{a}_{q}\right| /\left\|\boldsymbol{a}_{p}\right\|\left\|\boldsymbol{a}_{q}\right\|$.

Theorem 1. [16] CPD in (6) is essentially unique if coherences of factor matrices satisfy $\frac{1}{\mu_{A}}+\frac{1}{\mu_{B}}+\frac{1}{\mu_{S}} \geq 2 R+2$.

For space shift $\boldsymbol{B}$, coherence $\mu_{B}$ is a measure of the angular separation between sources, similarly to $\boldsymbol{A}^{\text {shift }}$, whereas the coherence $\mu_{S}$ of signal matrix $S$ is the largest correlation coefficient between sources. For pure gain pattern diversity embedded in $\boldsymbol{A}^{\text {gain }}$ of (5), coherence $\mu_{A}^{\text {gain }}$ is a measure of similarity among pattern responses to impinging sources. Since $\boldsymbol{a}_{p}^{\top} \boldsymbol{a}_{q}=\sum_{\ell=1}^{L} g_{\ell}\left(\theta_{p}\right) g_{\ell}\left(\theta_{q}\right), \frac{\left|\boldsymbol{a}_{p}^{\top} \boldsymbol{a}_{q}\right|}{\left\|\boldsymbol{a}_{p}\right\|\left\|\boldsymbol{a}_{q}\right\|} \leq 1$, with equality if and only if $g_{\ell}\left(\theta_{p}\right)=g_{\ell}\left(\theta_{q}\right) \forall \ell$.

Thus, the uniqueness condition stated in Th. 1 can be interpreted in a physical sense: the tensor model of $\S$ II-A1 is unique if sources are not too closely spaced, if their directivity response is not too similar, and if their time signatures are not too correlated.

\section{ESTIMATION OF SOURCES AND DoA}

The tensor model in (6) can be expressed in vector form as

$$
\boldsymbol{x}=\operatorname{vec}\{\boldsymbol{\mathcal { X }}\}=\sum_{r=1}^{R} \boldsymbol{s}_{r} \otimes \boldsymbol{b}_{r} \otimes \boldsymbol{a}_{r}+\boldsymbol{n}
$$

where $\nabla$ denotes the Kronecker product, as defined in [14], [17]. Since the measurement noise vector, $\boldsymbol{n}=\operatorname{vec}\{\mathcal{N}\}$, is circular white Gaussian and isotropic, i.e. with zero mean and covariance $\boldsymbol{\Sigma}=\sigma^{2} \boldsymbol{I}$, the $\log$-likelihood takes the form:

$$
\Upsilon(\boldsymbol{\vartheta}) \propto-(\boldsymbol{x}-\boldsymbol{\mu})^{\mathrm{H}} \boldsymbol{\Sigma}^{-1}(\boldsymbol{x}-\boldsymbol{\mu})
$$

where $\boldsymbol{\mu}=\sum_{r=1}^{R} \boldsymbol{s}_{r} \otimes \boldsymbol{b}_{r} \otimes \boldsymbol{a}_{r}$ is unknown and constrained by its actual parameterization $\boldsymbol{\vartheta}=[\boldsymbol{\theta}, \operatorname{vec}\{\boldsymbol{G}\}, \operatorname{vec}\{\boldsymbol{P}\}, \operatorname{vec}\{\boldsymbol{S}\}]$. The CRB represents the lower bound on the variance of

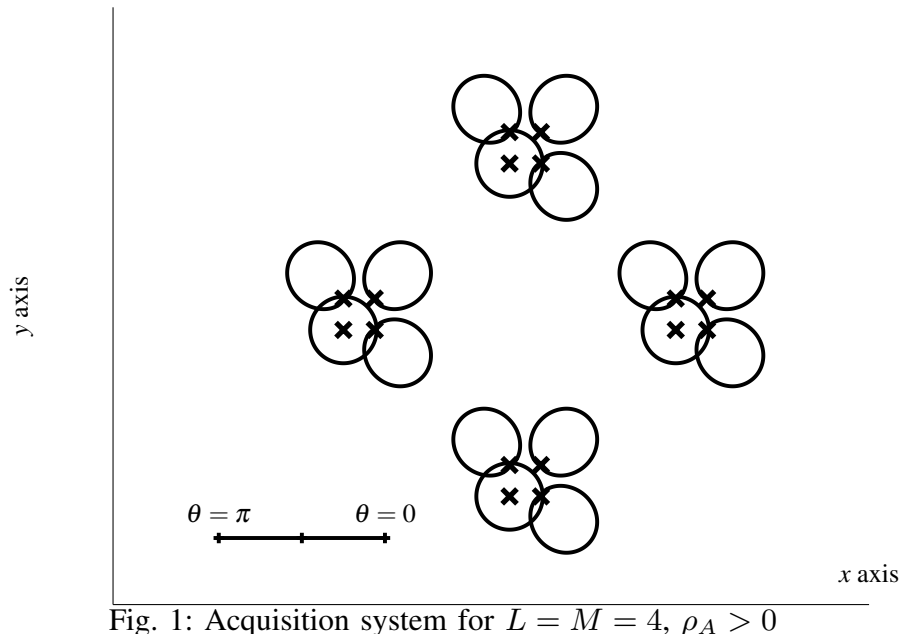

any unbiased estimator and is equal to the inverse of the Fisher Information Matrix (FIM). For the derivation of the FIM of the estimation problem in (8), refer to [11] and references therein. Similarly to [10], the CRB is computed assuming that $\boldsymbol{a}_{r}$ and $\boldsymbol{s}_{r}$ are nuisance parameters, i.e. with parameter vector $\chi$ composed of DoAs and factor matrices: $\boldsymbol{\chi}=[\boldsymbol{\theta}, \operatorname{vec}\{\boldsymbol{A}\}, \operatorname{vec}\{\boldsymbol{S}\}]$.

Although the optimal solution is given by the global maximum of $\Upsilon(\boldsymbol{\vartheta})$, we propose a suboptimal two-step procedure in the same spirit as [10], with smaller computational burden: first $\Upsilon$ is maximized with respect to parameter $\boldsymbol{\xi}=[\operatorname{vec}\{\boldsymbol{A}\}, \operatorname{vec}\{\boldsymbol{B}\}, \operatorname{vec}\{\boldsymbol{S}\}]$ through a CP decomposition routine, such as Alternating Least Squares (ALS) [18]; then DoAs can be estimated by

$$
\hat{\theta}_{r}=\arg \min _{\theta \in \Theta}\left[\left(\hat{\boldsymbol{b}}_{r}-\boldsymbol{b}_{r}(\theta)\right)^{\mathrm{H}}\left(\hat{\boldsymbol{b}}_{r}-\boldsymbol{b}_{r}(\theta)\right)\right] .
$$

This solution can then, if necessary, be refined by a local ascent of $\Upsilon(\boldsymbol{\vartheta})$. However, this improvement has revealed to be negligible in subsequent computer experiments.

\section{Computer Results}

For the sake of simplicity, sources and the acquisition system are assumed to be coplanar. Sensor positions then become $\boldsymbol{p}_{\ell}=\left[p_{\ell}^{x}, p_{\ell}^{y}\right], 1 \leq \ell \leq L$, and delays becomes $\tau_{\ell}\left(\theta_{r}\right)=\left[p_{\ell}^{x} \cos \left(\theta_{r}\right)+p_{\ell}^{y} \sin \left(\theta_{r}\right)\right] / c$, both functions of azimuth only. This amounts to considering elevation $\psi_{r}=\pi / 2, \forall r$. We work with UCAs of radius $\rho$, with $p_{\ell}^{x}=\rho \cos (2 \pi \ell / L)$ and $p_{\ell}^{y}=\rho \sin (2 \pi \ell / L)$, as in [5]. We choose a directivity pattern $g(\cdot)$ with maximum gain in the radial directions from the center of the array, $2 \pi \ell / L$. Hence, the response of sensor $\ell$ to source $r$ amounts to $g_{\ell}\left(\theta_{r}\right)=g\left(\theta_{r}-2 \pi \ell / L\right)$. Function $g(\theta)$ is chosen to be a simple nonnegative, smooth and $2 \pi$ periodical function, with

$$
g(\theta)^{2}=\frac{D(\gamma)}{2^{\gamma}}(1+\cos (\theta))^{\gamma}
$$

and parameter $\gamma$ controls directivity $D(\gamma)=\frac{2^{\gamma} 2 \pi}{\int_{0}^{2 \pi}(1+\cos (\theta))^{\gamma} d \theta}$ exponentially.

We simulated $R=4$ uncorrelated narrowband sources arriving from $\boldsymbol{\theta}=\left[25^{\circ}, 65^{\circ}, 105^{\circ}, 345^{\circ}\right]$ with $T=64$ time 


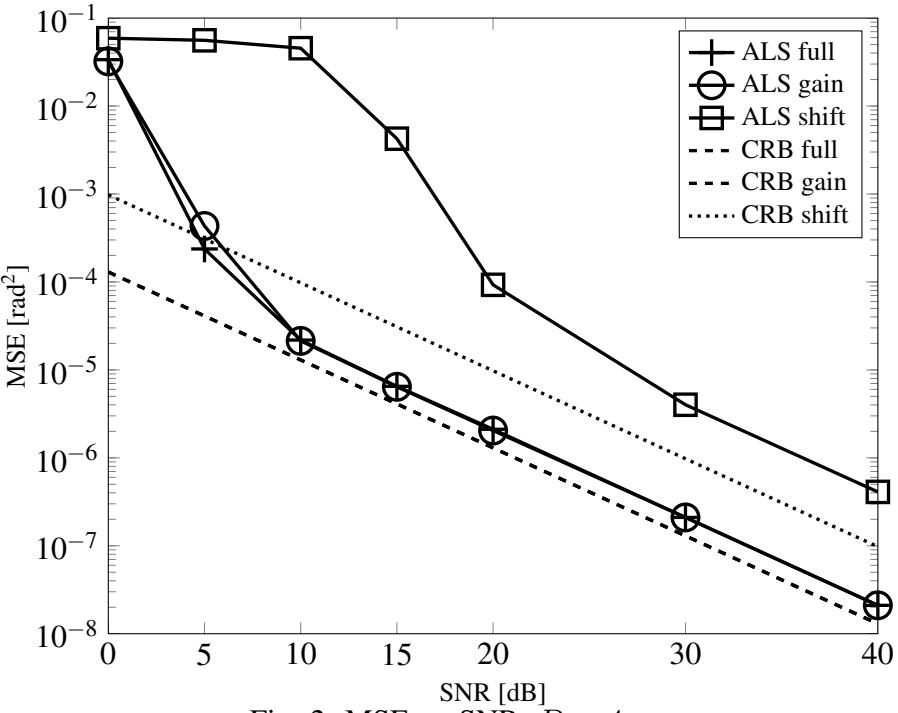

Fig. 2: MSE vs SNR, $D=4$

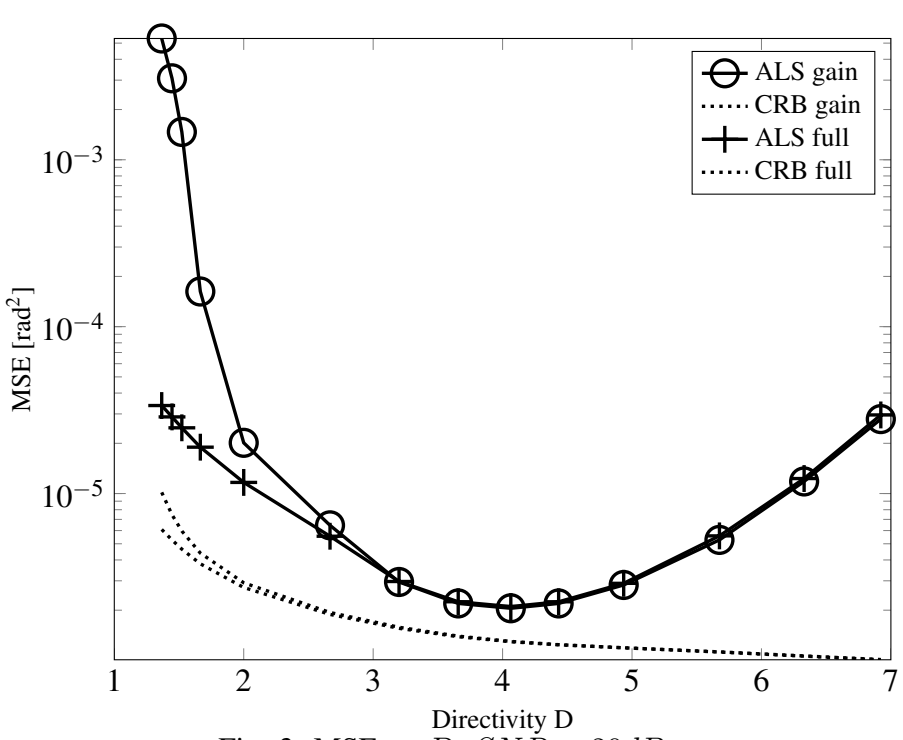

Fig. 3: MSE vs $D, S N R=20 d B$

samples. Each subarray is a UCA of radius $\rho_{A}=\lambda /(20 \sqrt{2})$ with $L=4$ sensors, whereas gain patterns have $D(\gamma)=4$ (i.e. $\gamma=5) . M=4$ subarrays with the aforementioned structure are located around a UCA of radius $\rho_{B}=\lambda /(2 \sqrt{2})$ (see Figure 1). As in [10], [19], SNR is defined as:

$$
S N R=10 \log _{10} \frac{\mathbb{E}\left[\boldsymbol{\mu}^{\mathrm{H}} \boldsymbol{\mu}\right]}{\mathbb{E}\left[\boldsymbol{n}^{\mathrm{H}} \boldsymbol{n}\right]}=10 \log _{10} \frac{\|\boldsymbol{\mu}\|_{2}^{2}}{L M T \sigma^{2}}
$$

and estimated Mean Square Error is defined as $\operatorname{MSE}(\boldsymbol{\theta})=\frac{1}{\pi^{2}} \frac{1}{N R} \sum_{n=1}^{N} \sum_{r=1}^{R}\left(\hat{\theta}_{r n}-\theta_{r}\right)^{2}$. Results are obtained by averaging over $N=200$ noise realizations.

The three approaches compared in Figures 2, 3, 4 refer to tensor DoA estimation, with the same equivalent SNR:

ALS full refers to tensor $\mathcal{M}^{\text {full }}$ in (4), when sensors are non overlapping $\left(\rho_{A}>0\right)$ and directional: $A_{\ell r}^{\text {full }}=$ $g_{\ell}\left(\theta_{r}\right) e^{-\jmath \omega_{0} \tau_{\ell}\left(\theta_{r}\right)}$ and $\sigma^{2 \text { full }}=\frac{\left\|\boldsymbol{\mu}^{f u l l}\right\|_{2}^{2}}{L M T} 10^{-S N R / 10}$.

$A L S$ gain refers to tensor $\mathcal{M}^{\text {gain }}$ in (5), when sensors are overlapping $\left(\rho_{A}=0\right)$ and directional: $A_{\ell r}^{\text {gain }}=g_{\ell}\left(\theta_{r}\right)$

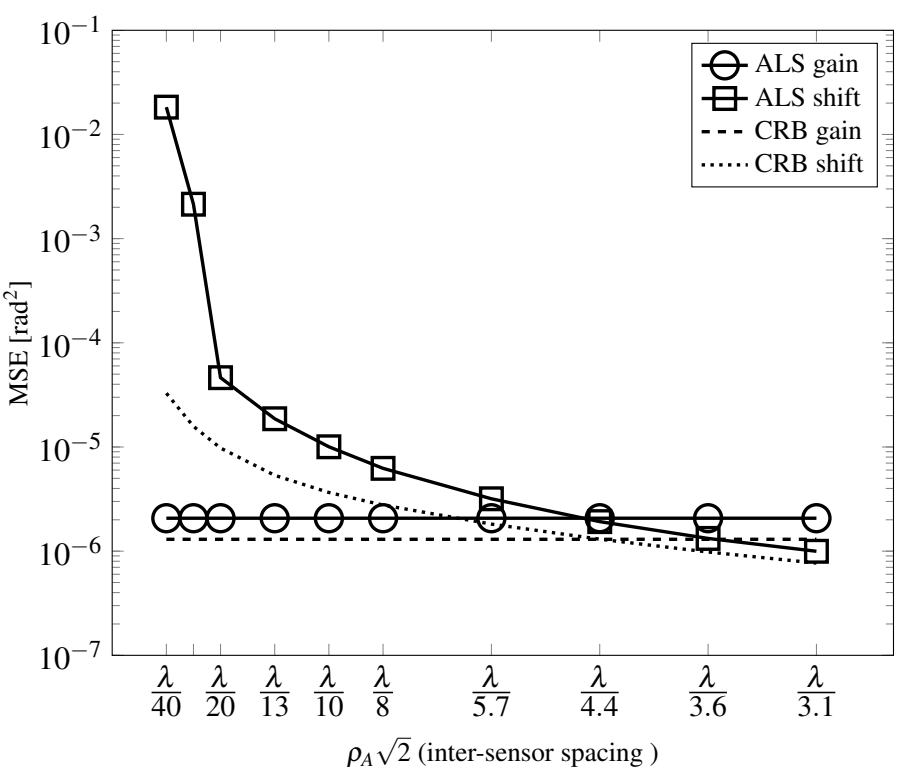

Fig. 4: MSE vs $\rho_{A} \sqrt{2}$ (inter-sensor distance), $S N R=20 d B, D=4$

and $\sigma^{2 \text { gain }}=\frac{\left\|\boldsymbol{\mu}^{\text {gain }}\right\|_{2}^{2}}{L M T} 10^{-S N R / 10}$.

$A L S$ shift refers to tensor $\mathcal{M}^{\text {shift }}$ as in [9], when sensors are non overlapping and omni-directional: $A_{\ell r}^{\text {shift }}=$ $e^{-\jmath \omega_{0} \tau_{\ell}\left(\theta_{r}\right)}$ and $\sigma^{2 \text { shift }}=\frac{\left\|\boldsymbol{\mu}^{\text {shift }}\right\|_{2}^{2}}{L M T} 10^{-S N R / 10}$.

Figure 2 shows MSE as a function of SNR: when sensor positions within the reference subarray are not known, the introduction of unknown directional elements improves the estimation ( $A L S$ full), even when sensors overlap (ALS gain).

Figure 3 illustrates the dependence of the MSE on sensor directivity $D$, showing an optimum at $D \approx 4$ (i.e. $\gamma \approx 5$ ) for the present configuration. The large value of the MSE for small and large directivity is due to bad conditioning of factor matrix $\boldsymbol{A}^{\text {gain }}$. Indeed, as $D$ grows gain patterns $g_{\ell}\left(\theta_{r}\right)$ become elongated toward one direction, thus attenuating all the others. In this configuration, pattern coherence $\mu_{A}$ approaches 1 .

Figure 4 shows the dependence of the MSE on the intersensor distance within the reference subarray, for ALS shift (omnidirectional non overlapping sensors, $\rho_{A}>0$ ) in comparison with ALS gain (directional overlapping sensors, null inter-sensor distance, $\rho_{A}=0$ ). The former is a decreasing function of inter-sensor distance, hence intersecting the latter at a critical distance where the presence of directional elements is not essential for tensor modeling.

\section{CONCLUSION}

We already knew from [9] that space, time, and translation in space induced exploitable diversities, when omnidirectional sensors are used. This remains true if sensors have known nonzero gain patterns, because they can be compensated. But the question whether sensor gain patterns could induce a diversity of their own was still open. We showed that it can indeed be the case, even when sensors are co-located within each subarray. In this case, there is no space diversity anymore, but tensor approaches, which need at least three diversities, can still be applied thanks to gain pattern diversity. 


\section{REFERENCES}

[1] W. Liu and S. Weiss, Wideband beamforming: concepts and techniques, vol. 17, John Wiley \& Sons, 2010.

[2] H. Krim and M. Viberg, "Two decades of array signal processing research: the parametric approach," IEEE Signal Process. Mag., vol. 13, no. 4, pp. 67-94, 1996.

[3] T. Rahim and D.E.N. Davies, "Effect of directional elements on the directional response of circular antenna arrays," IEE Proceedings $H$ Microw., Optics and Antennas, vol. 129, no. 1, pp. 18-22, 1982.

[4] M. Chryssomallis, "Smart antennas," IEEE Antennas and Propag. Mag., vol. 42, no. 3, pp. 129-136, 2000.

[5] B. R. Jackson, S. Rajan, B. J. Liao, and S. Wang, "Direction of arrival estimation using directive antennas in uniform circular arrays," IEEE Trans. Antennas Propag., vol. 63, no. 2, pp. 736-747, 2015.

[6] M. Pesavento and J.F. Bohme, "Direction of arrival estimation in uniform circular arrays composed of directional elements," in Proc. IEEE Workshop Sensor Array Multichannel Sig. Process., 2002, pp. 503-507.

[7] C. Chang, T. Cheng, and H. Lin, "Fast direction finding algorithm for circular array based on directional antenna," in Intern. Workshop on Microw. Millimeter Wave Circuits Syst. Technol. IEEE, 2012, pp. 1-4.

[8] R. O. Schmidt, "Multiple emitter location and signal parameter estimation," IEEE Trans. Antennas Propag., vol. 34, no. 3, pp. 276-280, Mar. 1986.

[9] N. D. Sidiropoulos, R. Bro, and G. B. Giannakis, "Parallel factor analysis in sensor array processing," IEEE Trans. Signal Process., vol. 48, no. 8, pp. 2377-2388, Aug. 2000.

[10] S. Sahnoun and P. Comon, "Joint source estimation and localization," IEEE Trans. Signal Process., vol. 63, no. 10, pp. 2485-2495, 2015.

[11] F. Raimondi, R. Cabral Farias, O. Michel, and P. Comon, "Wideband multiple diversity tensor array processing," Preprint, hal-01350549, Aug. 2016.

[12] R. Roy and T. Kailath, "ESPRIT-estimation of signal parameters via rotational invariance techniques," IEEE Trans. Acoust. Speech Signal Process., vol. 37, no. 7, pp. 984-995, 1989.

[13] A. L. Swindlehurst, B. Ottersten, R. Roy, and T. Kailath, "Multiple invariance ESPRIT," IEEE Trans. Signal Process., vol. 40, no. 4, pp. 867-881, 1992.

[14] P. Comon, "Tensors: a brief introduction," in IEEE Signal Process. Mag., 2014, vol. 31, pp. 44-53.

[15] L. Chiantini and G. Ottaviani, "On generic identifiability of 3-tensors of small rank," SIAM Journal on Matrix Analysis and Applications, vol. 33, no. 3, pp. 1018-1037, 2012.

[16] L.-H. Lim and P. Comon, "Blind multilinear identification," IEEE Trans. Inf. Theory, vol. 60, no. 2, pp. 1260-1280, Feb. 2014.

[17] T. G. Kolda and B. W. Bader, "Tensor decompositions and applications," SIAM Review, vol. 51, no. 3, pp. 455-500, Sept. 2009.

[18] P. Comon, X. Luciani, and A. L. F. De Almeida, "Tensor decompositions, alternating least squares and other tales," Journal of chemometrics, vol. 23, no. 7-8, pp. 393-405, 2009.

[19] X. Liu and N. D. Sidiropoulos, "Cramér-Rao lower bounds for lowrank decomposition of multidimensional arrays," IEEE Trans. Signal Process., vol. 49, no. 9, pp. 2074-2086, 2001. 